\title{
Co-regulation of intragenic microRNA miR-153 and its host gene Ia-2 $\beta$ : identification of miR-153 target genes with functions related to $I A-2 \beta$ in pancreas and brain
}

\author{
W. Mandemakers • L. Abuhatzira • H. Xu • \\ L. A. Caromile • S. S. Hébert • A. Snellinx • \\ V. A. Morais - S. Matta - T. Cai - A. L. Notkins • \\ B. De Strooper
}

Received: 23 January 2013 / Accepted: 26 February 2013 / Published online: 18 April 2013

(C) The Author(s) 2013. This article is published with open access at Springerlink.com

\begin{abstract}
Aims/hypothesis We analysed the genomic organisation of miR-153, a microRNA embedded in genes that encode two of the major type 1 diabetes autoantigens, isletassociated protein (IA)-2 and IA- $2 \beta$. We also identified
\end{abstract}

W. Mandemakers and L. Abuhatzira contributed equally to this study.

Electronic supplementary material The online version of this article (doi:10.1007/s00125-013-2901-5) contains peer-reviewed but unedited supplementary material, which is available to authorised users.

W. Mandemakers $\cdot$ S. S. Hébert · A. Snellinx $\cdot$ V. A. Morais ·

S. Matta $\cdot$ B. De Strooper

VIB Center for the Biology of Disease, Gasthuisberg O\&N4,

Herestraat 49-bus 602,

3000 Leuven, Belgium

W. Mandemakers $\cdot$ S. S. Hébert $\cdot$ A. Snellinx $\cdot$ V. A. Morais S. Matta $\cdot$ B. De Strooper

Center for Human Genetics, LIND and Universitaire

Ziekenhuizen, KULeuven, Leuven, Belgium

L. Abuhatzira $\cdot$ H. Xu $\cdot$ L. A. Caromile $\cdot$ T. Cai $\cdot$ A. L. Notkins Experimental Medicine Section, Oral Infection and Immunity Branch, National Institute of Dental and Craniofacial Research, National Institute of Health, Bethesda, MD, USA

B. De Strooper $(\square)$

Center for Human Genetics-CB4, VIB Center for the Biology of Disease-VIB11, Gasthuisberg O\&N4, Herestraat 49-bus 602, 3000 Leuven, Belgium

e-mail: bart.destrooper@cme.vib-kuleuven.be

\section{Present address:}

S. S. Hébert

Neurosciences, Centre de Recherche du CHUQ (CHUL),

RC-9800, 2705 boul. Laurier,

Québec, QC, Canada
miR-153 target genes that correlated with IA- $2 \beta$ localisation and function.

Methods A bioinformatics approach was used to identify miR-153's genomic organisation. To analyse the coregulation of miR-153 and IA- $2 \beta$, quantitative PCR analysis of miR-153 and $I a-2 \beta$ (also known as Ptprn2) was performed after a glucose stimulation assay in MIN6B cells and isolated murine pancreatic islets, and also in wild-type Ia-2 (also known as Ptprn), Ia-2 $\beta$ single knockout and Ia$2 / I a-2 \beta$ double knockout mouse brain and pancreatic islets. Bioinformatics identification of miR-153 target genes and validation via luciferase reporter assays, western blotting and quantitative PCR were also carried out.

Results Two copies of miR-153, miR-153-1 and miR-1532 , are localised in intron 19 of $I a-2$ and $I a-2 \beta$, respectively. In rodents, only miR-153-2 is conserved. We demonstrated that expression of miR-153-2 and $I a-2 \beta$ in rodents is partially co-regulated as demonstrated by a strong reduction of miR-153 expression levels in $I a-2 \beta$ knockout and Ia-2/Ia-2 $\beta$ double knockout mice. miR-153 levels were unaffected in $I a-2$ knockout mice. In addition, glucose stimulation, which increases $I a-2$ and $I a-2 \beta$ expression, also significantly increased expression of miR-153. Several predicted targets of miR-153 were reduced after glucose stimulation in vitro, correlating with the increase in miR-153 levels.

Conclusions/interpretation This study suggests the involvement of miR-153, IA-2 $\beta$ and miR-153 target genes in a regulatory network, which is potentially relevant to insulin and neurotransmitter release.

Keywords Diabetes · Glucose stimulation · IA-2 $\beta$. MicroRNA $\cdot \operatorname{miR}-153 \cdot$ Neurodegeneration 


$\begin{array}{ll}\text { Abbreviations } \\ \text { AKO } & I a-2 \text { single knockout } \\ \text { BKO } & I a-2 \beta \text { single knockout } \\ \text { DCV } & \text { Dense core vesicle } \\ \text { DKO } & I a-2 / I a-2 \beta \text { double knockout } \\ \text { IA } & \text { Islet-associated protein } \\ \text { IPA } & \text { Ingenuity Pathway Analysis } \\ \text { NIH } & \text { National Institutes of Health } \\ \text { miRNA } & \text { MicroRNA } \\ \text { Pri } & \text { Primary RNA transcript } \\ \text { PTP } & \text { Protein tyrosine phosphatase } \\ \text { SCR } & \text { Scrambled sequence } \\ \text { SNAP25 } & \text { Synaptosomal-associated protein } 25 \\ \text { SNCA } & \text { Alpha-synuclein } \\ \text { TSS } & \text { Transcription start site } \\ \text { UTR } & \text { Untranslated region } \\ \text { VAMP2 } & \text { Vesicle-associated membrane protein } 2\end{array}$

\section{Introduction}

Islet-associated protein (IA) 2 and IA- $2 \beta$ are major autoantigens in type 1 diabetes [1]. Based on sequence, IA-2 and IA- $2 \beta$, also known as ICA512 and phogrin, respectively, are members of the transmembrane protein tyrosine phosphatase (PTP) family, but are enzymatically inactive with standard PTP substrates because of two critical amino acid substitutions in the PTP domain [2]. However, recent studies have shown that IA- $2 \beta$ has low phosphatidylinositol phosphatase activity [3]. Both proteins consist of an intracellular, transmembrane and luminal domain and are produced in neuroendocrine cells throughout the body (e.g. pancreatic islets, brain) [4-8]. Knockout mice of the individual Ia-2 (also known as Ptprn) and Ia-2 $\beta$ (also known as Ptprn2) genes and Ia-2/Ia-2 $\beta$ double knockout (DKO) mice do not develop diabetes, but show abnormal glucose tolerance, impaired insulin secretion and reduced insulin content in beta cells [9-12]. Moreover, DKO mice display defects in neurotransmitter release (dopamine, noradrenaline [norepinephrine] and serotonin), resulting in behavioural and learning disturbances, seizures and reduced lifespan [13]. These findings demonstrate that IA-2 and IA$2 \beta$ not only affect the secretion of insulin, but also that of neurotransmitters, raising the possibility that defects in the IA-2-IA-2 $\beta$ pathway might not only lead to glucose intolerance, but also to neurological disorders.

MicroRNAs (miRNAs) function as a rheostat of the genome and proteome by controlling gene expression at the post-transcriptional level [14]. These short ( $\sim 21$ to 23 nucleotides [nt]), conserved, non-coding RNA molecules are transcribed from the genome as miRNA precursor molecules, which are processed to single stranded miRNA by a series of ribonucleases. The mature miRNA is integrated into the RNA-induced silencing complex (RISC) and binds partial complementary mRNA sequences that mostly reside in the $3^{\prime}$ untranslated region (UTR) of mRNA. Binding of the miRNA to its target mRNA results in either translational repression or mRNA destabilisation [15]. In mammals, about one thousand conserved miRNAs have been identified and can be localised as either intragenic (intronic or exonic) or intergenic [16]. Intragenic miRNAs can be transcriptionally regulated by their own regulatory elements and/or co-regulated with their host gene. A single miRNA can influence the translation of several mRNA sequences at once, potentially affecting parallel biological pathways. Dysregulation of miRNA expression contributes to various diseases, including cancer [17], and cardiovascular [18] and neurodegenerative diseases [19].

Examination of the genomic sequence of $I A-2$ (also known as $P T P R N$ ) and $I A-2 \beta$ (also known as PTPRN2) revealed that two miRNAs, miR-153-1 and miR-153-2, are embedded in these genes. Because of the importance of IA- 2 and IA- $2 \beta$ in type 1 diabetes, we investigated whether miR-153 expression is co-regulated with $I A-2$ and $I A-2 \beta$; we also determined whether it might be involved in regulating target genes that play a role in similar cellular functions to those affected by IA2 and IA- $2 \beta$, such as secretion of neurotransmitters in the brain and insulin release by beta cells in pancreas. This study provides the first evidence of a miR-153-IA-2 $\beta$-target gene regulatory pathway and sets the stage for further investigations to obtain a greater mechanistic insight into this pathway.

\section{Methods}

Animal care and use Targeted disruption of the individual $I a-2$ and $I a-2 \beta$ genes has been described previously [10-12, 20]. Because female $I a-2^{-/} / I a-2 \beta^{-/}$mice are infertile, male $\mathrm{Ia}-2^{-/-} / \mathrm{Ia}-2 \beta^{-/-}$mice were bred to female $\mathrm{Ia}-2^{+-} / \mathrm{Ia}-2 \beta^{-/-}$ mice to generate $I a-2^{-/} / I a-2 \beta^{-/}$(DKO) mice. $I a-2^{+/+} / I a-$ $2 \beta^{+/+}$wild-type mice served as controls. Animal studies were conducted under protocols approved by the Institutional Animal Care and Use Committees of the USA at the National Institutes of Health (NIH).

Reagents Cell culture reagents were purchased from Invitrogen (Ghent, Belgium). Antibodies were derived from the following sources: mouse anti- $\beta$-actin (A5441; SigmaAldrich [Diegem, Belgium]), mouse anti-alpha-synuclein (SNCA) (610787; BD-Biosciences [Erembodegem, Belgium]), mouse anti-parkin (PARK2) (MAB5512; Millipore [Overijsse, Belgium]), rabbit anti-synaptosomal-associated protein 25 (SNAP25) (AB1762; Millipore) and mouse antibassoon (monoclonal antibody SAP7F407; Enzo Life Sciences, [Antwerpen, Belgium]). Total RNA from mouse pancreas, heart and brain was purchased from Biochain 
(Kampenhout, Belgium). Total RNA and miRNA from wild-type and DKO mouse islets were extracted using a kit (miRNeasy Mini kit; Qiagen [Antwerpen, Belgium]) according to the manufacturer's protocols.

Cell lines MIN6B mouse insulinoma cell line (ATCC, Molsheim, France) was cultured at $37^{\circ} \mathrm{C}$ in $95 \%$ air and $5 \%$ $\mathrm{CO}_{2}$ in DMEM supplemented with $15 \%$ vol./vol. heatinactivated fetal bovine serum, $2 \mathrm{mmol} / \mathrm{l}$ L-glutamine, $100 \mathrm{U} / \mathrm{ml}$ penicillin and $0.1 \mathrm{mg} / \mathrm{ml}$ streptomycin. The human neuroblastoma SH-SY5Y cell line (ATCC) was cultured in DMEM/F12 medium supplemented with $10 \%$ vol./vol. fetal bovine serum, $100 \mathrm{U} / \mathrm{ml}$ penicillin and $100 \mu \mathrm{g} / \mathrm{ml}$ streptomycin; the culture process was at $37^{\circ} \mathrm{C}$ in $95 \%$ air and $5 \% \mathrm{CO}_{2}$.

Glucose-stimulated gene expression assay in fresh mouse islets Islets from 3- to 4-month-old sex-matched mice were isolated as previously described [21], with slight modifications according to the manufacturer's protocol for Collagenase P (Roche, Indianapolis, IN, USA), and stimulated with various concentrations of glucose, followed by RNA extraction and quantitative PCR analysis. See electronic supplementary material (ESM) Methods for further details.

Glucose-stimulated gene expression assay in MIN6B cells MIN6B cells were stimulated with various concentrations of glucose, followed by RNA extraction and quantitative PCR analysis. See ESM Methods for further details.

Quantitative RT-PCR (mRNA) Total RNA was extracted using a kit (mirVana Paris kit; Applied Biosystems [Ghent, Belgium]) and following the manufacturer's instructions. RT-PCR was performed with a high-capacity cDNA reverse transcription kit (Applied Biosystems) according to the manufacturer's instructions; for quantitative PCR we used the Taqman gene expression assay (Applied Biosystems) for $I a-2, I a-2 \beta$, primary RNA transcript (pri) pri-miR-153 (Mm03306386_pri), Snca, Syt1, Syt4, Bsn, Pclo, Vamp2, Snap25, Gapdh, Tbp and $\beta$-actin according to the manufacturer's instructions and using a LightCycler 480 Real-Time PCR System (Roche, Basel, Switzerland).

Quantitative RT-PCR (miRNA) Total RNA was extracted using a kit (mirVana Paris kit; Applied Biosystems) and following the manufacturer's instructions. Each RT-PCR reaction was performed in triplicate. The Taqman miRNA reverse transcription kit (Applied Biosystems) and the Taqman Universal PCR master mix (Applied Biosystems) were used according to the manufacturers' instructions. The quantitative PCR procedures were carried out following the instructions provided with the Taqman miRNA assays (Applied Biosystems). The following miRNA assay was used: mmu-miR-153 (Applied Biosystems). Relative miR-153 expression was calculated by using the comparative cycle threshold method and normalised to the following reference genes: RNU19 (also known as Snora74a), Sno135 (also known as Snord65) and miR-16.

Bioinformatics identification of potential miR-153 targets involved in neurotransmitter and insulin release Analysis of co-production of IA-2 $\beta$ and predicted miR-153 targets (predicted by Targetscan www.targetscan.org release 6.2, accessed June 2012; 629 top predicted conserved targets) in brain and pancreas was performed by searching the literature. Overlap of IA-2 $\beta$ and miR-153 target gene expression was further validated using the following online gene expression databases: BIOGPS (http://biogps.gnf.org, accessed Sept 2012), the type 1 diabetes database (T1Dbase) (www.t1dbase.org, accessed Sept 2012) and the Allan brain atlas (www.brain-map.org/, accessed Sept 2012) (data not shown). Targets that met these criteria were used for further analysis to determine possible protein $\times$ protein interactions and gene-function relationships between the predicted targets using Ingenuity Pathways Analysis (IPA) 9.0 (Ingenuity Systems, www.ingenuity.com, accessed Sept 2010).

Protein extraction and western blot analysis Cultured cells were rinsed with cold PBS and lysed in the cell disruption buffer from the mirVANA Paris kit (Applied Biosystems). Note that with this extraction procedure, co-isolation of proteins and high-quality RNA (including the small RNA species) from the same sample is possible, allowing direct comparative measurements (of miRNAs, mRNA and protein) within a given tissue or cells. This protocol was used to extract RNA and proteins from SH-SY5Y cells, and from adult wild-type, $I a-2$ single knockout (AKO), Ia- $2 \beta$ single knockout (BKO) and DKO mouse brains. Proteins were analysed by western blotting according to standard procedures. See ESM Methods for further details.

Transfections, DNA cloning and luciferase assay MIN6B and SH-SY5Y cells were transfected using a transfection reagent (HiPerFect; Qiagen) or Lipofectamine 2000 (Invitrogen) according to the manufacturers' instructions and further processed for quantitative PCR analysis or luciferase reporter assays. See ESM Methods for further details.

\section{Results}

Localisation and co-regulation of miR-153 and IA-2/IA-2 $\beta$ genes Genomic localisation of many mammalian miRNA transcription units has been determined previously [22]. Using these data and the University of California Santa Cruz (UCSC) genome browser (http://genome.ucsc.edu/cgi-bin/ hgTracks, accessed Sept 2012), we determined that two 
copies of the miR-153 small non-coding miRNAs exist in the human genome. In humans, miR-153-1 is localised at a highly conserved region in intron 19 of $I A-2$ on chromosome 2 (chr2:219862589-219882387) (Fig. 1a). miR-153-2 is localised at a highly conserved intronic region between exon 19 and 20 of $I A-2 \beta$ on chromosome 7 (chr7:157059789-157059875) (Fig. 1a). Next, we used ClustalW multiple alignment software (www.genome.jp/ tools/clustalw/) to determine whether miR-153-1 and miR153-2 are evolutionarily conserved (Fig. 1b). We found that although miR-153-2 is evolutionarily highly conserved, miR-153-1 is more divergent. In particular, in rodents (mouse, rat), polymorphisms are localised within the seed sequence of miR-153-1. This makes it very unlikely that functional miR-153 is generated from the miR-153-1 transcription unit in rodents. This was validated by performing a predicted hybridisation analysis using the RNAhybrid algorithm [23] and SNCA 3' UTR as a confirmed miR-153 target [24] (ESM Fig. 1). These data indicate that, based on the strong evolutionary conservation of miR-153-2 within the intron of the $I A-2 \beta$ gene, miR-153-2 and $I A-2 \beta$ might be functionally connected and co-regulated.

To investigate whether expression of miR-153-2 and $I A-$ $2 \beta$ are co-regulated, we determined whether the $I A-2 \beta$ mRNA and the pri-miR-153 transcript are generated from the same or separate heterogeneous nuclear (hn)RNA transcripts. We therefore first analysed the expression of mir153 in brain material of wild-type, $\mathrm{AKO}, \mathrm{BKO}$ and DKO mice. $\mathrm{AKO}$ and $\mathrm{BKO}$ mice were generated previously, resulting in a complete loss of $I a-2$ and $I a-2 \beta$ mRNA expression and protein production $[10,12]$. The knockout strategy does not target the intron in which miR-153-2 is located. Quantitative PCR measurements showed that miR-153 expression was reduced in BKO and DKO, but not in wild-type or AKO mouse brain material after normalisation to three independent small non-coding RNA controls (miR-16 [also known as Gde1], RNU19 [also known as Snora74a], Sno135; only results for the latter are shown) (Fig. 2a). To rule out the possibility that the observed remaining miR-153 was generated by cross-hybridisation of the oligonucleotides with the miR-153-1 sequences in the $I a-2$ locus, we analysed expression of the primiR-153-2 product, which only results in the amplification of the miR-153-2, but not of the miR-153-1 product. We showed that pri-miR-153 expression was reduced to the same extent as that of mature miR-153 in BKO and DKO, but not in wild-type and AKO mouse brain RNA (Fig. 2b). Interestingly, pri-miR153 expression was reduced to almost undetectable levels in isolated DKO islets compared with wild-type (Fig. 2c).

Next, we analysed mirR-153 expression in mouse MIN6B cells and isolated mouse pancreatic islets after glucose stimulation (Fig. 2d,e). We detected a significant increase in the expression of miR-153, Ia-2 and $I a-2 \beta$ after glucose stimulation of MIN6B cells at $25 \mathrm{mmol} / \mathrm{l}$ glucose compared with $5 \mathrm{mmol} / \mathrm{l}$ glucose (Fig. 2d). Finally, increasing

$\mathbf{a}$

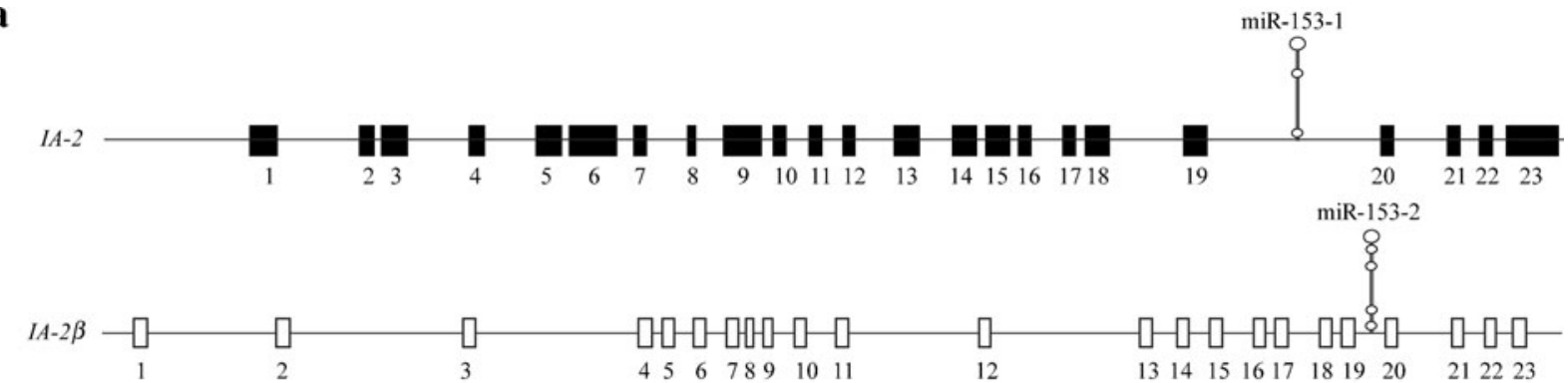

b

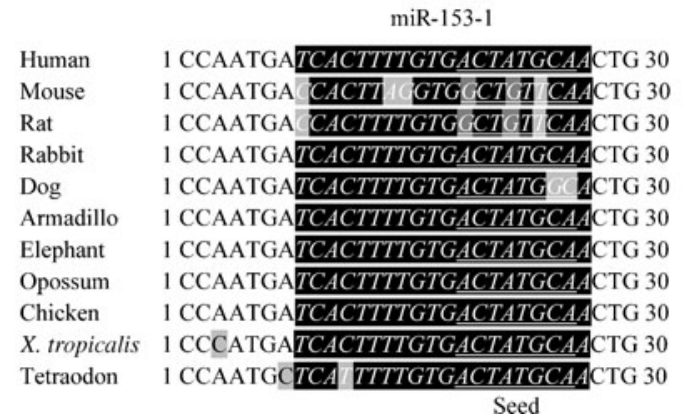

miR-153-2

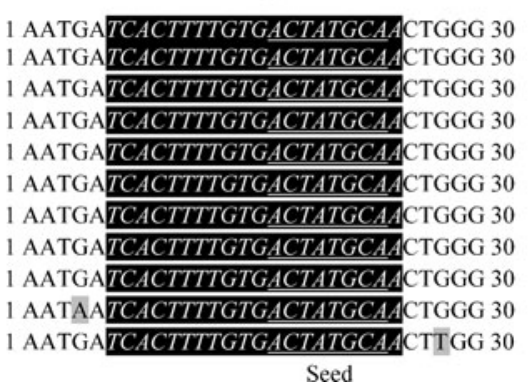

Fig. 1 Genomic organisation of miR-153 transcription unit. (a) Schematic representation of the $I A-2$ and $I A-2 \beta$ genomic loci (not to scale). The locations of miR-153-1 and miR-153-2 are indicated (between exon 19 and exon 20). The $I A-2$ gene in humans and mice extends over $20 \mathrm{~kb}$ and consists of 23 exons. The genomic structure of $I A-2 \beta$ in humans and mice consists of 23 exons and spans approximately 1,000 and $800 \mathrm{~kb}$, respectively. (b) Evolutionary conservation of miR-153-1 and miR-153-2 sequences as determined by ClustalW alignment. Seed sequences are underlined. Black or grey shading indicates conserved or nonconserved residues, respectively. X. tropicalis, Xenopus tropicalis 

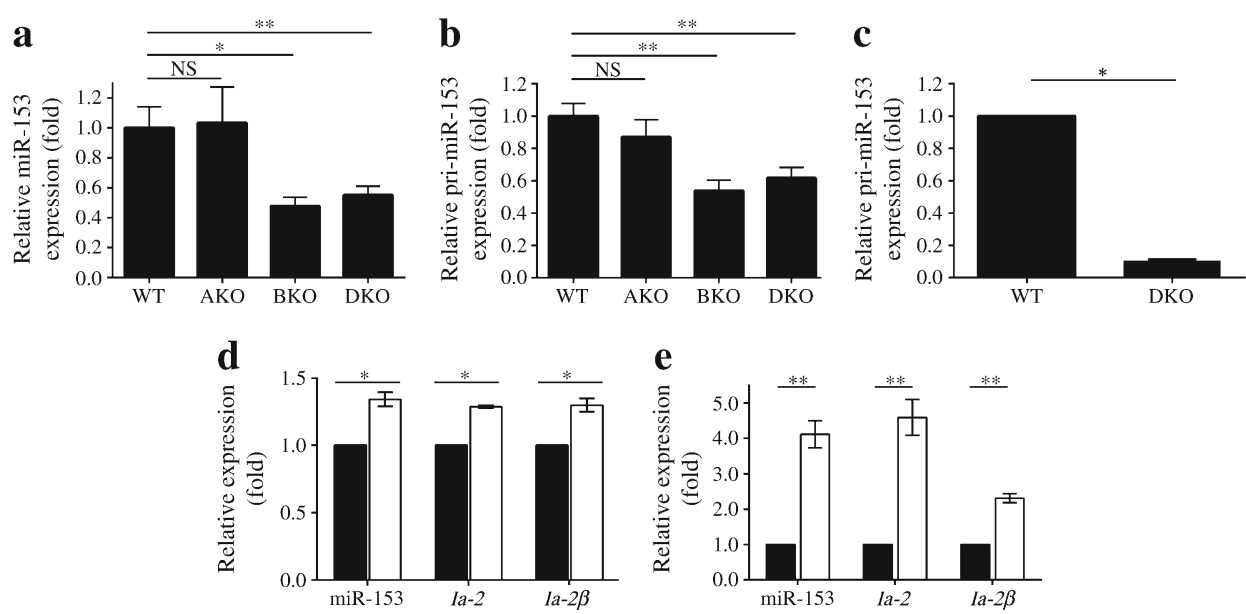

Fig. 2 Co-regulation of miR153 and Ia-2 $\beta$ expression. (a) Quantitative PCR analysis of miR-153 mRNA expression in wild-type (WT), $\mathrm{AKO}, \mathrm{BKO}$ and DKO mouse brain. Relative expression (fold) was calculated using the relative cycle threshold quantification method $\left(2^{-\Delta \Delta \mathrm{C}_{\mathrm{t}}}\right)$ and normalised to Sno135; WT $n=6$, AKO $n=4$, BKO $n=4$, DKO $n=6$. (b) Quantitative PCR analysis of pri-miR-153 mRNA expression in mice as above (a). Relative expression (fold) was calculated as above (a) and normalised to Tbp; WT $n=6$, AKO $n=4$, BKO $n=4$, DKO $n=6$. (c) Quantitative PCR analysis of pri-miR-153 mRNA expression in wild-type and DKO mouse pancreatic islets. Relative expression (fold) was calculated as above (a) and normalised to Tbp;

WT $n=6$, DKO $n=6$. (d) Quantitative PCR analysis of Ia-2, Ia-2 $\beta$ and miR-153 transcript levels in cultures of MIN6B cells at $5 \mathrm{mmol} / 1$ (black bars) and $25 \mathrm{mmol} / \mathrm{l}$ (white bars) glucose stimulation. Relative expression (fold) was calculated as above (a) and normalised to $\beta$-actin. $n=3$. (e) Quantitative PCR analysis of $I a-2, I a-2 \beta$ and miR-153 transcript levels in pancreatic islets from adult wild-type $(\mathrm{C} 57 \mathrm{BL} / 6)$ mice upon stimulation with $3.3 \mathrm{mmol} / \mathrm{l}$ (black bars) and $16.7 \mathrm{mmol} / \mathrm{l}$ (white bars) glucose. Relative expression (fold) was calculated as above (a) and normalised to $\beta$-actin; $n=6$. Experiments $(\mathbf{a}-\mathbf{e})$ were performed in triplicate and significance determined by Mann-Whitney $U$ test, ${ }^{*} p<0.05$ and ${ }^{* *} p<0.01$

glucose levels from 3.3 to $16.7 \mathrm{mmol} / 1$ dramatically increased miR-153, Ia-2 and $I a-2 \beta$ expression in cultured isolated mouse islets (Fig. 2e). These studies show that miR-153, Ia2 and $I a-2 \beta$ expression is increased after glucose stimulation in MIN6B cells and isolated mouse pancreatic islets.

These results also show that whereas miR-153 expression was almost completely lost in DKO islets (Fig. 2c), miR-153 levels in DKO brain were still detectable at about $50 \%$ of the levels found in wild-type mouse brain (Fig. 2b). This might indicate that in addition to miR-153 being generated from a transcript and transcription start site (TSS) shared with the host gene $I a-2 \beta$, an alternative tissue-specific transcript and TSS for miR-153 might exist independently of its host gene $I a-2 \beta$. Moreover, the targeted $I a-2 \beta$ allele was generated by deleting its promoter region [10]. Thus, loss of the $I a-2 \beta$ promoter rules out the possibility that any remaining miR-153 expression is generated from shared regulatory elements in the promoter region of its host gene, $I a-2 \beta$. We performed an in silico analysis of putative miR-153-2 TSSs in humans, using a miRStart database search [25]. A putative miR153-2 TSS was identified at 38,909 bp from the start of the miR-153 precursor (ESM Fig. 2). Further experimental evidence, outside the scope of the current study, would be required to determine whether this is a conserved and tissue-specific miR-153 TSS.

Bioinformatics identification of potential miR-153 targets involved in neurotransmitter and insulin release Many

potential miR-153 targets can be determined by various miRNA target-prediction software programs (data not shown). However, only a few miR-153 targets have been experimentally validated [24, 26-31]. Although it is generally accepted that miRNA-mRNA co-expression leads to inhibition of target protein levels [15], several reports indicate that this correlation is less apparent in postnatal brain, where miRNA expression can be associated with the presence rather than absence of target protein expression [32, 33]. Furthermore, it has previously been shown that intronic miRNAs can regulate the expression of their target genes, which can play a role in the same functional regulatory pathway as the host gene in which the miRNA is located $[34,35]$. Based on these findings and our observation that miR-153-2 and $I A-2 \beta$ expression are co-regulated, we can narrow down the list of candidate miR-153 targets based on its co-regulation with $I A-2 \beta$ expression. Predicted targets of miR-153 and potential protein regulatory networks regulated by miR-153 have previously been identified [24]. Using bioinformatics analysis (see Methods), we set out to refine this list of potential miR-153 targets as defined by correlation with $I A-2 \beta$ expression patterns (brain and pancreas) and IA-2 $\beta$ function (i.e. synaptic vesicle and dense core vesicle $[\mathrm{DCV}]$ release). From this analysis we extracted target genes that are highly associated with $I A-2 \beta$ expression and function (ESM Table 1). Next, we used IPA software to determine gene-function relationships (Table 1) and potential interactions (Fig. 3a) between IA-2 $\beta$, miR-153 and the 
Table 1 Gene-function relationships between miR-153 target genes
The top 20 relevant biological functions are given

The $p$ values were calculated using the right-tailed Fisher's Exact Test. The smaller the $p$ value, the less likely a random association and the more significant the association

${ }^{\mathrm{a}}$ Names of proteins involved in each predicted biological function are abbreviated; BSN, bassoon; PCLO, piccolo; PTPRN2, protein tyrosine phosphatase, receptor type, $\mathrm{N}$ polypeptide 2; SYT, synaptotagmin; VAMP2, vesicle-associated membrane protein 2

\begin{tabular}{lll}
\hline Relevant biological function & $p$ value & Molecules $^{\mathrm{a}}$ \\
\hline Fusion of synaptic vesicles & $3.40 \times 10^{-12}$ & BSN, SNAP25, SYT1, VAMP2 \\
Secretion of neurotransmitter & $4.21 \times 10^{-12}$ & PTPRN2, SNAP25, SNCA, SYT1, SYT4, VAMP2 \\
Transport of synaptic vesicles & $5.63 \times 10^{-12}$ & PCLO, SNCA, SYT1, SYT4, VAMP2 \\
Exocytosis & $2.48 \times 10^{-11}$ & PCLO, SNAP25, SNCA, SYT1, SYT4, VAMP2 \\
Exocytosis by cells & $2.43 \times 10^{-10}$ & PCLO, SNAP25, SNCA, SYT1, VAMP2 \\
Release of neurotransmitter & $5.64 \times 10^{-10}$ & PCLO, SNAP25, SNCA, SYT1, SYT4, VAMP2 \\
Excitatory postsynaptic potential & $4.20 \times 10^{-8}$ & SNAP25, SNCA, SYT1, VAMP2 \\
Presence of fusion pores & $1.13 \times 10^{-7}$ & SYT1, SYT4 \\
Quantity of synaptic vesicles & $1.82 \times 10^{-7}$ & PCLO, PTPRN2, SNCA \\
Miniature excitatory postsynaptic & $3.00 \times 10^{-7}$ & SNAP25, SYT1, VAMP2 \\
currents & & \\
Synthesis of neurotransmitter & $6.70 \times 10^{-7}$ & SNAP25, SNCA, SYT1 \\
Exocytosis of vesicles & $7.10 \times 10^{-7}$ & SNAP25, SYT1, VAMP2 \\
Flux of noradrenaline & $2.38 \times 10^{-6}$ & SYT1, SYT4 \\
Synaptic transmission & $6.55 \times 10^{-6}$ & BSN, SNAP25, SNCA, SYT1 \\
Exocytosis of synaptic vesicles & $3.96 \times 10^{-5}$ & SYT1, VAMP2 \\
Secretion of dopamine & $4.58 \times 10^{-5}$ & PTPRN2, SNCA \\
Secretion of L-glutamic acid & $5.95 \times 10^{-5}$ & PTPRN2, SNCA \\
Exocytosis of granules & $7.09 \times 10^{-5}$ & SYT4, VAMP2 \\
Endocytosis & $1.64 \times 10^{-4}$ & SNAP25, SNCA, SYT1 \\
Fusion of cellular membrane & $1.92 \times 10^{-4}$ & SYT4, VAMP2 \\
\hline
\end{tabular}

a

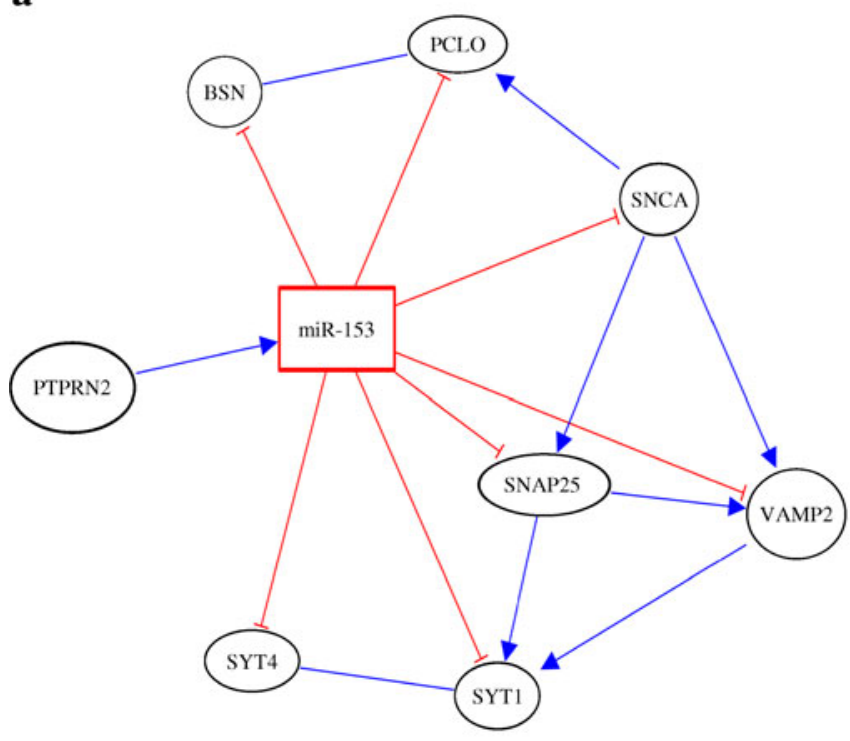

b
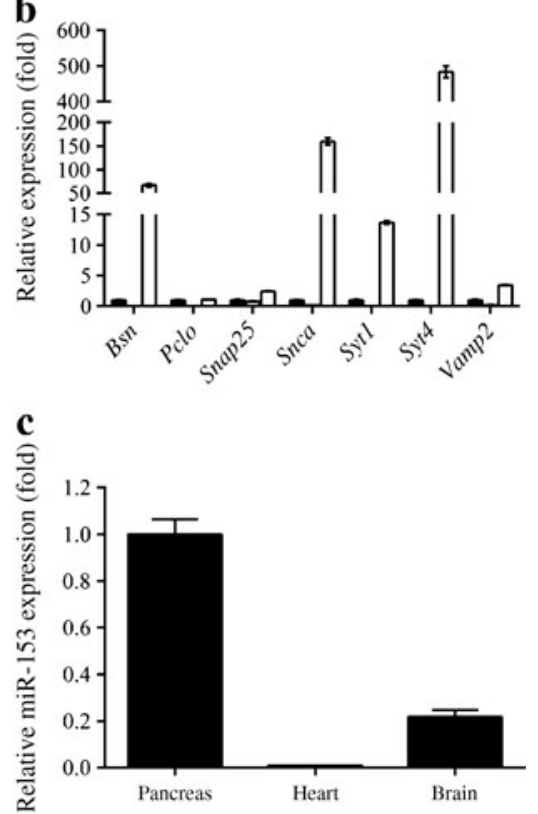

Fig. 3 miR-153 target gene prediction analysis. (a) Predicted protein $\times$ protein interactions using IPA 9.0. Various points of interaction between the candidate miR-153 targets are shown. The arrow from protein tyrosine phosphatase, receptor type, $\mathrm{N}$ polypeptide 2 (PTPRN2, also known as IA-2 $\beta$ ) to miR-153 indicates that PTPRN2 affects the abundance of miR-153. Red lines with a short line at the end indicate an inhibitory effect of miRNA target gene interaction. Lines between two proteins indicate that only binding was detected. Blue arrows indicate the direction of protein $\times$ protein interaction. BSN, bassoon; PCLO, piccolo; SYT, synaptotagmin. (b) Expression of predicted targets as indicated was determined by quantitative PCR on adult wild-type mouse total RNA from pancreas (black bars), heart (grey bars) and brain (white bars). (c) Expression of pri-miR-153 was determined by quantitative PCR in adult wild-type (C57BL/6) mouse total RNA from pancreas (black bar), heart (grey bar) and brain (white bar). (b,c) Relative expression (fold) of miR-153 was calculated using the relative quantification method, taking mouse pancreas levels as calibrator, i.e. onefold. All data were normalised to Tbp in three independent experiments $(n=4)$ 
selected human candidate miR-153 target genes. We also showed that the predicted target genes and miR-153 are expressed in pancreas and brain as determined by quantitative PCR (Fig. 3b,c). Our data support a significant association of miR-153 and its target genes with vesicle exocytosis $[24,36-38]$. Based on these findings, the identified candidates are very likely to be regulated by miR- 153 .

Analysis of regulation of predicted miR-153 target genes in vivo and in vitro Based on our data showing that miR-153 expression is significantly reduced in mouse pancreatic islets (Fig. 2c) and to a lesser extent in brains from DKO mice (Fig. 2b), we hypothesised that the expression of predicted target genes would be increased in DKO islets and brains. To test this, we examined the mRNA expression of predicted miR-153 targets in DKO islets and BKO mouse brains (Fig. 4a,b). In islets we found a significant increase in Pclo, Snap25 and Vamp2 expression (Fig. 4a), whereas only Bsn expression was significantly increased in DKO mouse brain (Fig. 4b). Although most other targets followed a trend towards increased expression, they did not show statistically significant changes. Next, based on our data showing that miR-153 expression was induced after glucose stimulation in MIN6B cells (Fig. 2d), we hypothesised that the expression of predicted target genes would be reduced after glucose stimulation if they are directly regulated by miR-153 action. To analyse this possibility, we used quantitative PCR to investigate target gene expression in glucose-stimulated MIN6B cells (Fig. 4c). Here we found that Bsn expression was significantly reduced (Fig. 4c), whereas most other targets, although showing a trend towards reduced expression, did not change significantly. Subsequently, to determine whether overexpression of miR-153 in mouse MIN6B or in human neuroblastoma SHSY5Y cells leads to reduced target gene expression, we transfected these cells either with miR-153 pre-miR precursor or a scrambled control precursor (SCR) (both at $50 \mathrm{nmol} / 1$ final concentration) and analysed target gene expression at $48 \mathrm{~h}$ after transfection. miR-153 expression was analysed by quantitative PCR analysis to determine the extent of miR-153 overexpression
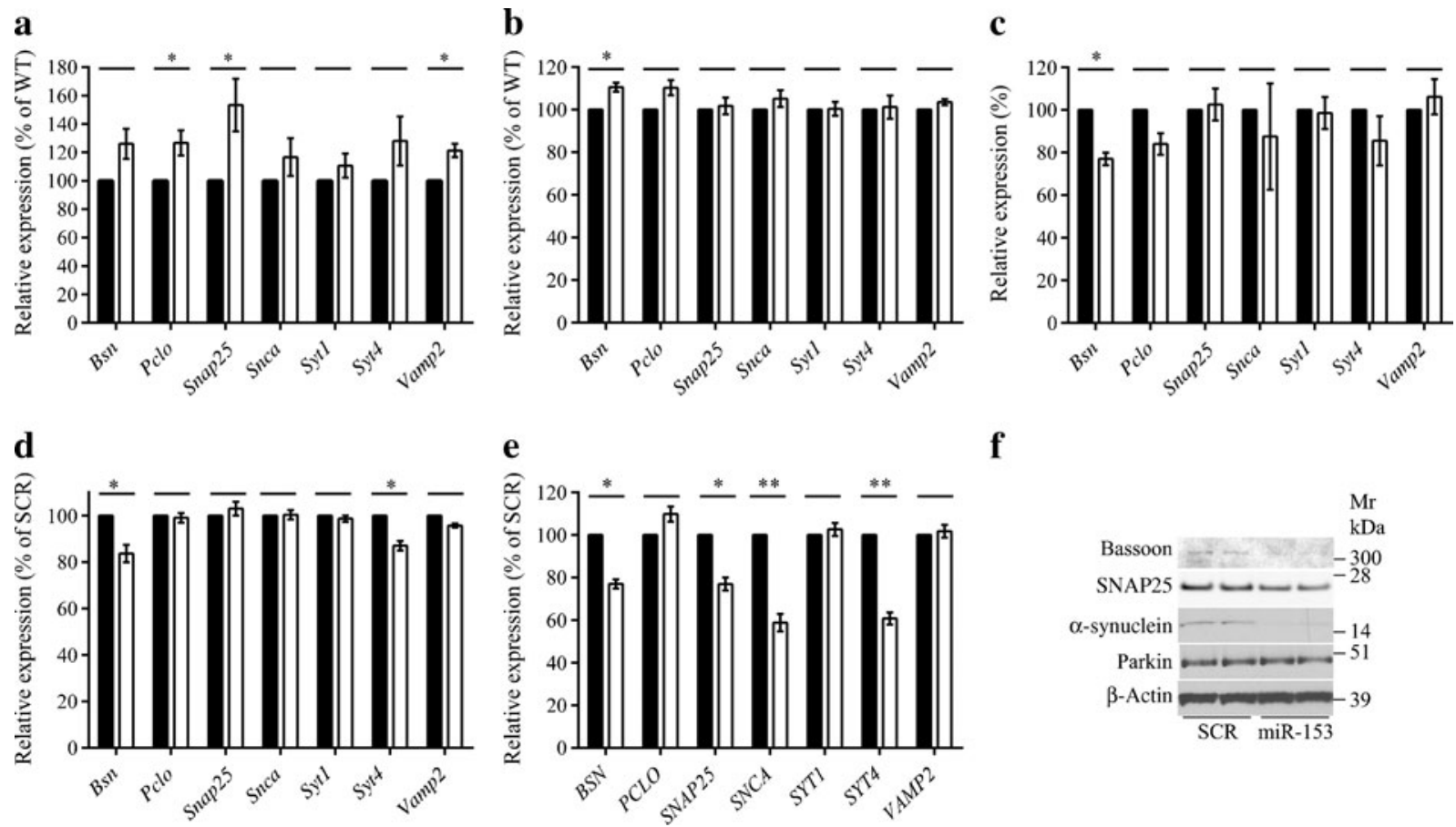

f

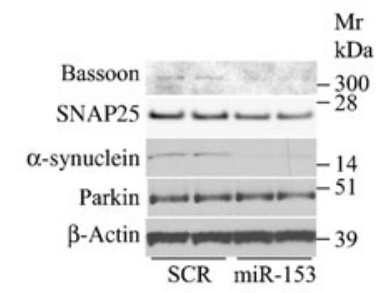

Fig. 4 miR-153 regulation of $S N C A$ expression in vitro and expression of miR-153 targets after glucose stimulation and in $I a-2 \beta$ knockout mice. (a) Gene expression analysis of predicted miR-153 target genes as determined by quantitative PCR in pancreatic islets of wild-type (black bars) and Ia-2/Ia-2 $\beta$ knockout (white bars) mice or (b) in brains of wild-type (black bars) and Ia-2 $\beta$ knockout (white bars) mice. The relative expression (as percentage of wild-type) of miR-153 targets was calculated using the relative quantification method (taking the level of wild-type for each target gene as calibrator, i.e. set at 100\%) and normalised to Tbp. (c) Gene expression analysis as above $(\mathbf{a}, \mathbf{b})$ in MIN6B cells in response to $5 \mathrm{mmol} / \mathrm{l}$ (black bars) or $25 \mathrm{mmol} / \mathrm{l}$ (white bars) glucose stimulation. The relative expression of miR-153 targets (as percentage of $5 \mathrm{mmol} / \mathrm{l}$ glucose treatment) was calculated using the

relative quantification method (with $5 \mathrm{mmol} / \mathrm{l}$ glucose for each target gene as calibrator, i.e. set at one) and normalised to Tbp. (d) Gene expression analysis as above $(\mathbf{a}, \mathbf{b})$ in MIN6B cells or (e) SH-SY5Y cells after induction of miR-153 precursor or SCR overexpression. The relative expression of miR-153 targets (as percentage of SCR treatment) was calculated using the relative quantification method and normalised to Tbp. (a-e) Error bars represent SEM; $n>3$ independent duplicate experiments. Significance was determined using the MannWhitney $U$ test, $* p<0.05$ and $* * p<0.01$. (f) Western blot analysis of endogenous bassoon, SNAP25, alpha-synuclein (SNCA), parkin and $\beta$-actin in SH-SY5Y cells treated with $50 \mathrm{nmol} / 1$ (final concentration) of miR-153 or SCR 
(ESM Fig. 3). We found significantly reduced expression of $B s n$ and Syt4 in mouse MIN6B cells (Fig. 4d), and significantly reduced BSN, SNAP25, SNCA and SYT4 expression in human SH-SY5Y cells (Fig. 4e). Next, we asked whether miR-153 can regulate endogenous protein levels of these predicted targets in SH-SY5Y cells. We used protein extracts from the same transfected cells used for the quantitative PCR analysis (Fig. 4e). We analysed the abundance of bassoon, SNAP25 and SNCA in SH-SY5Y cells, and showed that miR-153 overabundance downregulated bassoon abundance by $83 \%$, SNAP 25 by $43 \%$ and SNCA by $78 \%$ compared with scrambled control transfected cells (Fig. 4f). Protein levels of parkin and the housekeeping gene $\beta$-actin, which do not have any predicted miR153 seed sequences, remained unaffected by miR-153 overabundance.

Finally, to determine whether the predicted targets can be directly regulated by miR-153, we selected SNCA, bassoon and piccolo for further analysis. SNCA expression has been shown to be regulated by miR-153 [24], and both SNCA and IA- $2 \beta$ localise to secretory granules in beta cells, as well as to synaptic vesicles in neurons $[13,39]$. We confirmed that SNCA expression is regulated by miR-153 in vitro (Fig. 4e,f). Regulation of bassoon and piccolo levels by miR-153 has not been investigated. The human $S N C A$, $B S N$ and PCLO 3' UTRs (wild-type) or 3' UTR with a mutated miR-153 seed sequences (seed mutant) were cloned after the stop codon of Firefly luciferase under control of the human thymidine kinase promoter in the pGL3 expression construct (ESM Fig. 4) and co-transfected in SH-SY5Y cells with miR-153 or a negative control SCR (pre-miR precursor; $50 \mathrm{nmol} / \mathrm{l}$; Ambion [Ghent, Belgium]). Co-transfection of miR-153 and luciferase constructs produced $35 \%, 80 \%$ and $40 \%$ reductions of Firefly luciferase activity in luciferase reporter constructs containing the 3' UTRs of SNCA, $B S N$, and $P C L O$, respectively (Fig. 5). Mutation of the miR-153 seed sequence (seed mutant) abolished the inhibitory effect of miR-153 on luciferase activity in luciferase reporters of SNCA, and BSN. The human PCLO 3' UTR contains two miR-153-binding sites (ESM Fig. 4). Only mutation of the B seed sequence abolished the inhibitory effect of miR-153 on the PCLO 3' UTR luciferase reporter. These data show that miR-153 directly interacts with predicted seed sequences in SNCA, BSN and PCLO 3' UTRs to inhibit translation of the luciferase reporter protein levels.

These data indicate that changes in miR-153 abundance in various settings can result in significant changes of predicted target gene expression.

\section{Discussion}

This study describes the localisation of miR-153-1 and miR$153-2$ in introns of the human $I A-2$ and $I A-2 \beta$ genomic loci,

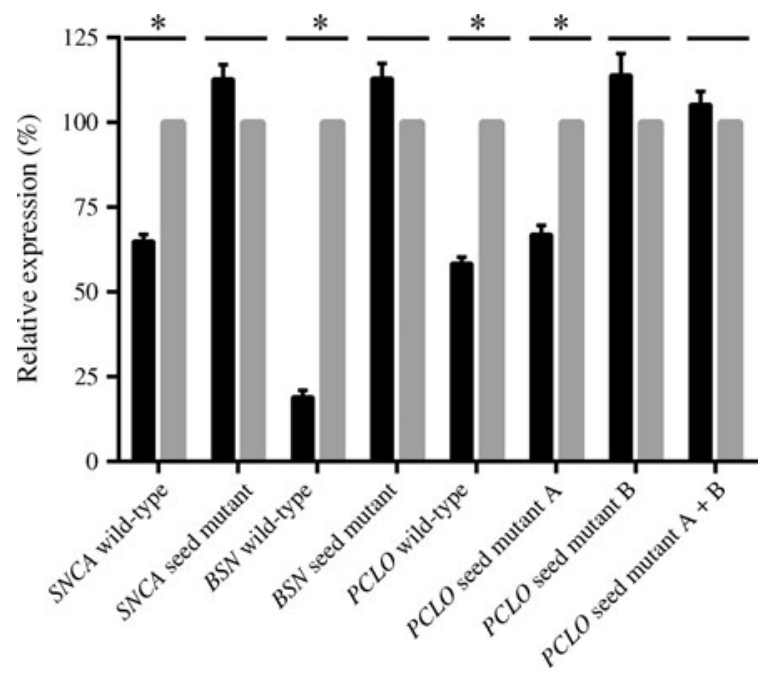

Fig. 5 Luciferase reporter analysis of predicted miR-153 targets. Luciferase and Renilla luciferase constructs were transfected into SHSY5Y cells with the miR-153 (black bars) or SCR (grey bars) oligonucleotides at a final concentration of $50 \mathrm{nmol} / \mathrm{l}$. Wild-type or seed mutant $S N C A, B S N$ and $P C L O 3^{\prime}$ UTR construct signals (see ESM Fig. 4, luciferase activity normalised to Renilla) are shown as a percentage of the signal in control cells transfected with SCR. Error bars represent SEM; $n>3$ independent triplicate experiments. Significance was determined using Mann-Whitney $U$ test, ${ }^{*} p<0.05$

respectively. We show that only miR-153-2 is evolutionarily conserved and partly co-regulated with $I A-2 \beta$ expression, as loss of $I a-2 \beta$ expression in BKO mice results in a strong reduction of miR-153 expression. We identified several potential miR-153 targets that correlate with $I A-2 \beta$ expression and function. We found significant changes in mRNA expression of several predicted target genes after glucose stimulation of MIN6B cells, as well as in isolated mouse pancreatic islets, where endogenous miR-153 levels are increased, and in BKO mouse brain and isolated islets, which have reduced miR-153 levels. Moreover, significant reductions of bassoon, SNAP25 and SNCA abundance in SH-SY5Y cells was found upon overproduction of miR-153 (Fig. 4f). It should be noted that in vivo miRNAs mainly function as rheostats or act via neutral repression, rather than being a binary off-switch to dampen protein output $[15,40]$.

The first point that arises from our study is that although in BKO mice mRNA expression of $I a-2 \beta$ is completely lost [10], reduced levels of miR-153 can still be detected in BKO and DKO brain, whereas miR-153 expression in DKO islets is almost completely lost (Fig. 2b, c). Interestingly, the effect of loss of miR-153 on predicted target gene expression appears to be more pronounced in isolated mouse knockout islets than in mouse knockout brains (Fig. 4a,b), correlating with the stronger reduction of miR-153 levels in knockout islets than in knockout brain. Moreover, the targeted $I a-2 \beta$ allele was generated by deleting its promoter region [10]. Thus, loss of the $I a-2 \beta$ promoter rules out the possibility that 
any remaining miR-153 expression that was detected in BKO and DKO mice was generated from shared regulatory elements in the promoter region of its host gene, $I a-2 \beta$, thus predicting the presence of an independent and tissuespecific miR-153 promoter region. Using miRStart software [25], we were able to detect putative transcription regulatory elements for miR-153-2 (ESM Fig. 2). Another study has also predicted a promoter region for miR-153-2 independently of the $I a-2 \beta$ TSS [41]. However, a recent study failed to find evidence that human miR-153-2 has a classical TSS, but instead contains a $\mathrm{CpG}$ island and transcription factor binding sites that could initiate transcription [42]. Interestingly, demethylation of methylated cytosines in two identified miR-153-2 CpG islands increases miR-153 expression [26]. Based on these studies and our findings, it is clear that miR-153-2 transcription unit can be regulated independently of $I a-2 \beta$ expression in a tissue-specific manner, but more detailed studies will be required to elucidate the exact transcriptional regulation of miR-153-2 expression.

Based on our present findings, earlier studies [9-13] that described the use of material from BKO or DKO mice should be judged with some caution, as it is possible that (some of) the observed results could have been caused by loss of miR-153 expression and potential upregulated expression of their targets. This issue probably needs to be considered in general for other studies that have generated targeted deletion in genes that contain intragenic miRNAs.

The present study points to the existence of a potential miR-153-IA-2 $\beta$-target gene pathway, which is involved in vesicle release in brain and pancreas. Indeed, it has already been shown that IA- $2 \beta$ localises to synaptic vesicles and that loss of IA- $2 \beta$ results in decreased dopamine release in PC12 cells $[13,43]$. Furthermore, DKO mice display defects in neurotransmitter release (dopamine, noradrenaline and 5-hydroxytryptamine (5-HT)) [13]. Various targets of miR153 have the same localisation and function in the same pathways as IA- $2 \beta$. For example, both SNCA and IA- $2 \beta$ localise to DCVs of beta cells, where loss of SNCA increases insulin release and loss of IA- $2 \beta$ decreases insulin release $[9,39]$. In addition, SNCA is a presynaptic protein that regulates synaptic vesicle dynamics and trafficking, and physically interacts with at least three other predicted miR153 target genes, i.e. Vamp2, Pclo and Snap25, to stimulate soluble n-ethyl maleimide sensitive factor (NSF) attachment protein receptor (SNARE) complex assembly [44]. Interestingly, mild overabundance of SNCA in mice resulted in a marked reduction of neurotransmitter release [45], similar to the phenotype observed in BKO mice [13]. Moreover, it has been shown that miR-153 expression is reduced in synaptosomes in comparison with the expression in total brain [32, 46, 47]. Finally, miR-153-1 has been associated with one of the type 1 diabetes mellitus loci (IDDM13), indicating that miR-153 may be a susceptibility candidate for human type 1 diabetes [48]. Taken together, the presence of miR-153-2 in the Ia-2 $\beta$ gene locus could be part of a feedback loop including miR-153 targets, and possibly regulating release of neurotransmitters at synapses and insulin secretion by beta cells. This possibility underscores the importance of the IA- $2 \beta-$ miR-153target gene pathway in brain and pancreas, and sets the stage for further investigations to obtain a greater mechanistic insight into this pathway.

Acknowledgements We thank G. Carmona (National Institute of Dental and Craniofacial research/NIH, Bethesda, MD, USA) for technical help.

Funding This work was supported, in part, by the Intramural Research Program of the National Institute of Dental and Craniofacial Research, $\mathrm{NIH}$, Bethesda, MD, USA, a Marie Curie Intra-European Fellowship (EIF proposal number 041333) awarded to W. Mandemakers, and a Methusalem grant (Flemish community and University of Leuven, Belgium) and European Research Commission (ERC) grant (EU Commission) to B. De Strooper.

Duality of interest The authors declare that there is no duality of interest associated with this manuscript

Contribution statement WM, LA, HX, LAC, SSH, VAM, SM, TC, ALN and BDS conceived and designed the experiments. WM, LA, HX, LAC, SSH and AS performed the experiments. All authors analysed the data. WM, LA, ALN and BDS were responsible for drafting the paper. All authors revised and approved the final version of paper.

Open Access This article is distributed under the terms of the Creative Commons Attribution Noncommercial License which permits any noncommercial use, distribution, and reproduction in any medium, provided the original author(s) and the source are credited.

\section{References}

1. Notkins AL, Lernmark A (2001) Autoimmune type 1 diabetes: resolved and unresolved issues. J Clin Invest 108:1247-1252

2. Magistrelli G, Toma S, Isacchi A (1996) Substitution of two variant residues in the protein tyrosine phosphatase-like PTP35/ IA-2 sequence reconstitutes catalytic activity. Biochem Biophys Res Commun 227:581-588

3. Caromile LA, Oganesian A, Coats SA, Seifert RA, Bowen-Pope DF (2010) The neurosecretory vesicle protein phogrin functions as a phosphatidylinositol phosphatase to regulate insulin secretion. J Biol Chem 285:10487-10496

4. Kawakami T, Saeki K, Takeyama N et al (2007) Detection of proteolytic cleavages of diabetes-associated protein IA-2 beta in the pancreas and the brain using novel anti-IA-2 beta monoclonal antibodies. Int J Mol Med 20:177-185

5. Roberts C, Roberts GA, Lobner K et al (2001) Expression of the protein tyrosine phosphatase-like protein IA-2 during pancreatic islet development. J Histochem Cytochem 49:767-776

6. Solimena M, Dirkx R Jr, Hermel JM et al (1996) ICA 512, an autoantigen of type I diabetes, is an intrinsic membrane protein of neurosecretory granules. EMBO J 15:2102-2114 
7. Takeyama N, Ano Y, Wu G et al (2009) Localization of insulinoma associated protein 2, IA-2 in mouse neuroendocrine tissues using two novel monoclonal antibodies. Life Sci 84:678-687

8. Wasmeier C, Hutton JC (1996) Molecular cloning of phogrin, a protein-tyrosine phosphatase homologue localized to insulin secretory granule membranes. J Biol Chem 271:18161-18170

9. Cai T, Hirai H, Zhang G et al (2011) Deletion of Ia-2 and/or Ia2beta in mice decreases insulin secretion by reducing the number of dense core vesicles. Diabetologia 54:2347-2357

10. Kubosaki A, Gross S, Miura J et al (2004) Targeted disruption of the IA-2beta gene causes glucose intolerance and impairs insulin secretion but does not prevent the development of diabetes in NOD mice. Diabetes 53:1684-1691

11. Kubosaki A, Nakamura S, Notkins AL (2005) Dense core vesicle proteins IA-2 and IA-2beta: metabolic alterations in double knockout mice. Diabetes 54(Suppl 2):S46-S51

12. Saeki K, Zhu M, Kubosaki A, Xie J, Lan MS, Notkins AL (2002) Targeted disruption of the protein tyrosine phosphatase-like molecule IA-2 results in alterations in glucose tolerance tests and insulin secretion. Diabetes 51:1842-1850

13. Nishimura T, Kubosaki A, Ito Y, Notkins AL (2009) Disturbances in the secretion of neurotransmitters in IA-2/IA-2beta null mice: changes in behavior, learning and lifespan. Neuroscience 159:427-437

14. Ambros V (2004) The functions of animal microRNAs. Nature 431:350-355

15. Bartel DP (2009) MicroRNAs: target recognition and regulatory functions. Cell 136:215-233

16. Chiang HR, Schoenfeld LW, Ruby JG et al (2010) Mammalian microRNAs: experimental evaluation of novel and previously annotated genes. Genes Dev 24:992-1009

17. Garofalo M, Croce CM (2011) MicroRNAs: master regulators as potential therapeutics in cancer. Annu Rev Pharmacol Toxicol $51: 25-43$

18. Small EM, Olson EN (2011) Pervasive roles of microRNAs in cardiovascular biology. Nature 469:336-342

19. Hebert SS, de Strooper B (2009) Alterations of the microRNA network cause neurodegenerative disease. Trends Neurosci 32:199-206

20. Kubosaki A, Nakamura S, Clark A, Morris JF, Notkins AL (2006) Disruption of the transmembrane dense core vesicle proteins IA-2 and IA-2beta causes female infertility. Endocrinology 147:811-815

21. Ravier MA, Rutter GA (2010) Isolation and culture of mouse pancreatic islets for ex vivo imaging studies with trappable or recombinant fluorescent probes. Methods Mol Biol 633:171-184

22. Rodriguez A, Griffiths-Jones S, Ashurst JL, Bradley A (2004) Identification of mammalian microRNA host genes and transcription units. Genome Res 14:1902-1910

23. Kruger J, Rehmsmeier M (2006) RNAhybrid: microRNA target prediction easy, fast and flexible. Nucleic Acids Res 34:W451W454

24. Doxakis E (2010) Post-transcriptional regulation of alphasynuclein expression by mir-7 and mir-153. J Biol Chem 285:12726-12734

25. Chien CH, Sun YM, Chang WC et al (2011) Identifying transcriptional start sites of human microRNAs based on high-throughput sequencing data. Nucleic Acids Res 39:9345-9356

26. Bao B, Rodriguez-Melendez R, Zempleni J (2011) Cytosine methylation in miR-153 gene promoters increases the expression of holocarboxylase synthetase, thereby increasing the abundance of histone H4 biotinylation marks in HEK-293 human kidney cells. J Nutr Biochem 23:635-639

27. Delay C, Calon F, Mathews P, Hebert SS (2011) Alzheimerspecific variants in the $3^{\prime} \mathrm{UTR}$ of amyloid precursor protein affect microRNA function. Mol Neurodegener 6:70
28. Liang C, Zhu H, Xu Y et al (2012) MicroRNA-153 negatively regulates the expression of amyloid precursor protein and amyloid precursor-like protein 2. Brain Res 1455:103-113

29. Long JM, Ray B, Lahiri DK (2012) MicroRNA-153 physiologically inhibits expression of amyloid-beta precursor protein in cultured human fetal brain cells and is dysregulated in a subset of Alzheimer disease patients. J Biol Chem 287:31298-31310

30. Xu J, Liao X, Lu N, Liu W, Wong CW (2011) Chromatinmodifying drugs induce miRNA-153 expression to suppress Irs-2 in glioblastoma cell lines. Int J Cancer 129:2527-2531

31. Xu J, Liao X, Wong C (2010) Downregulations of B cell lymphoma 2 and myeloid cell leukemia sequence 1 by microRNA 153 induce apoptosis in a glioblastoma cell line DBTRG-05MG. Int J Cancer 126:1029-1035

32. Schratt G (2009) microRNAs at the synapse. Nat Rev Neurosci 10:842-849

33. Tsang J, Zhu J, van Oudenaarden A (2007) MicroRNA-mediated feedback and feedforward loops are recurrent network motifs in mammals. Mol Cell 26:753-767

34. He C, Li Z, Chen P, Huang H, Hurst LD, Chen J (2012) Young intragenic miRNAs are less coexpressed with host genes than old ones: implications of miRNA-host gene coevolution. Nucleic Acids Res 40:4002-4012

35. Lutter D, Marr C, Krumsiek J, Lang EW, Theis FJ (2010) Intronic microRNAs support their host genes by mediating synergistic and antagonistic regulatory effects. BMC Genomics 11:224

36. Chi SW, Zang JB, Mele A, Darnell RB (2009) Argonaute HITSCLIP decodes microRNA-mRNA interaction maps. Nature 460:479-486

37. Liu H, Kohane IS (2009) Tissue and process specific microRNAmRNA co-expression in mammalian development and malignancy. PLoS One 4:e5436

38. Tsang JS, Ebert MS, van Oudenaarden A (2010) Genome-wide dissection of microRNA functions and cotargeting networks using gene set signatures. Mol Cell 38:140-153

39. Geng X, Lou H, Wang J et al (2011) alpha-Synuclein binds the K(ATP) channel at insulin-secretory granules and inhibits insulin secretion. Am J Physiol Endocrinol Metab 300:E276-E286

40. Ebert MS, Sharp PA (2012) Roles for microRNAs in conferring robustness to biological processes. Cell 149:515-524

41. Fujita S, Iba H (2008) Putative promoter regions of miRNA genes involved in evolutionarily conserved regulatory systems among vertebrates. Bioinformatics 24:303-308

42. Monteys AM, Spengler RM, Wan J et al (2010) Structure and activity of putative intronic miRNA promoters. RNA 16:495-505

43. Nishimura T, Harashima S, Yafang H, Notkins AL (2010) IA-2 modulates dopamine secretion in PC12 cells. Mol Cell Endocrinol 315:81-86

44. Burre J, Sharma M, Tsetsenis T, Buchman V, Etherton MR, Sudhof TC (2010) Alpha-synuclein promotes SNARE-complex assembly in vivo and in vitro. Science 329:1663-1667

45. Nemani VM, Lu W, Berge V et al (2010) Increased expression of alpha-synuclein reduces neurotransmitter release by inhibiting synaptic vesicle reclustering after endocytosis. Neuron 65:66-79

46. Pichardo-Casas I, Goff LA, Swerdel MR et al (2012) Expression profiling of synaptic microRNAs from the adult rat brain identifies regional differences and seizure-induced dynamic modulation. Brain Res 1436:20-33

47. Saba R, Storchel PH, Aksoy-Aksel A et al (2012) Dopamineregulated microRNA MiR-181a controls GluA2 surface expression in hippocampal neurons. Mol Cell Biol 32:619-632

48. Zhou L, He H, Mi JX, Li C, Lee B, Mi QS (2008) MicroRNA genes. Ann N Y Acad Sci 1150:72-75 